\title{
The conduction mechanism of nitronyl-nitroxide molecular magnetic compounds
}

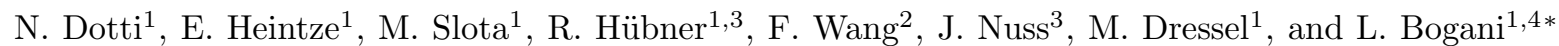 \\ ${ }^{1}$ 1.Physikalisches Institut, Universität Stuttgart, Pfaffenwaldring 57, D-70550, Stuttgart, Germany \\ ${ }^{2}$ Polymer and Materials Chemistry, Lund University, Box 124, SE-22100 Lund, Sweden \\ ${ }^{3}$ Max Plank Institute für Festkörperforschung, Heisenbergstraße 1, D-70569, Stuttgart, Germany. \\ ${ }^{4}$ Department of Materials, University of Oxford, 16 Parks Road, Oxford OX1 3PH, UK.
}

(Dated: December 21, 2015)

\begin{abstract}
We investigate the conduction mechanisms of nitronyl-nitroxide molecular radicals, as useful for the creation of nanoscopic molecular spintronic devices, finding that it does not correspond to standard Mott behaviour, as previously postulated. We provide a complete investigation using transport measurements, low-energy, sub- $\mathrm{THz}$ spectroscopy and introducing differently-substituted phenyl appendages. We show that a non-trivial surface-charge limited regime is present in addition to the standard low-voltage ohmic conductance. Scaling analysis allows determining all the main transport parameters for the compounds, and highlights the presence of charge trapping effects. Comparison among the different compounds shows the relevance of intermolecular stacking between the aromatic ring of the phenyl appendix and the NIT motif in the creation of useful electron transport channels. The importance of intermolecular pathways is further highlighted by electronic structure calculations, which clarify the nature of the electronic channels and their effect on the Mott character of the compounds.
\end{abstract}

PACS numbers: 75.50.Xx, 72.20.-i, 72.80.Le, 73., 78.

\section{INTRODUCTION}

Molecular magnetic materials offer a particularly rich ${ }^{57}$ experimental ground, ${ }^{1}$ where sophisticated effects can be observed with particular clarity and tuned by the means of molecular synthetic chemistry. ${ }^{2-7}$ They have 59 allowed, for example the observation of magnetic quan- 60 tum tunnelling, ${ }^{8-11}$ Berry phase interference, ${ }^{12}$ and per- 61 mit controlling the spin degrees of freedom by light ${ }^{13-20}{ }_{62}$ or via other external stimuli. ${ }^{21-25}$ Recent work has high- 63 lighted in particular the relevance of molecular magnetic ${ }^{64}$ materials for spintronics. ${ }^{26}$ A new area, described as 65 molecular spintronics, ${ }^{27-34}$ has arisen, where the molec- 66 ular magnetic properties can be used to tune the trans- 67 port properties of electronic devices. This approach has 68 different advantages: the chemical tunability of the mag- 69 netic molecules allows precise manipulation of the prop- 70 erties of spintronic systems, the electronic channels can ${ }^{71}$ be tuned by using rational chemical design, and the cre- 72 ated molecules can be integrated into nanoelectronic de- 73 vices, such as those formed by graphene ${ }^{35-38}$ and carbon 74 nanotubes, ${ }^{39-42}$ or onto functional surfaces. ${ }^{43-47}$

In this picture stable organic radicals constitute ${ }^{76}$ an appealing class of compounds because they pos- ${ }^{77}$ sess unpaired electrons, while being completely organic, and they are attracting rapidly-increasing attention for 78 molecular spintronics, ${ }^{48-50}$ switchable devices ${ }^{51-54}$ and 79 batteries. ${ }^{55-62}$ For all these purposes radicals are now be- 80 ing integrated into functional nanostructures, ${ }^{63-66}$ where 81 their spin and electric properties can be exploited. ${ }^{67}$ Moreover, organic radicals have attracted attention, 83 since the early days of molecular magnetism, as pos- 84 sible constituents for purely organic ferro-magnets, ${ }^{68}$ or for the creation of molecular magnetic coordination 86 compounds, ${ }^{69-74}$ including single molecule magnets and 87 single chain magnets. ${ }^{69}$ The study of the conduction mechanisms in bulk radicals is thus important as a comparison for nanostructured systems and for future investigations on radical-based molecular spintronics.

Electrically, organic radicals are usually classified as Mott insulators, ${ }^{75}$ because of the strong on-site Coulomb repulsions that hinder charge transport, despite the presence of singly-occupied molecular orbitals. At the same time, in-depth investigations have shown the presence of several intermolecular interaction channels, sometimes strong enough to transmit very sizeable magnetic interactions. $^{76}$ As the magnetic interaction is mediated by the electronic states of the molecules, these channels could, in principle, also be used to facilitate electronic transport in the material. Other transport channels, such as space charge limited (SCL) currents, ${ }^{77-80}$ could become relevant for the conduction of such systems, and the possible presence of charge traps ${ }^{81-84}$ also remain to be clarified. The identification of these elements and the possible rationalization of the structure-properties relation would be of fundamental importance for the design and development of spintronic nanodevices that rely on radicals.

Here we investigate the conduction properties of molecular radicals of the nitronyl nitroxide (NIT-R) family ${ }^{85,86}$ that contain an aromatic $\mathrm{R}$ appendage attached to the NIT group, by a combination of theoretical modeling, very low- energy (sub-THz) and high-voltage transport measurements, as never attempted before. We show that the systems do not follow the standard Mott behaviour considered so far, and, present characteristic channels that can be tuned chemically and can be exploited in perspective nanoscale devices. 


\section{EXPERIMENTAL METHODOLOGY}

We chose to investigate a family of NIT-R radicals containing different aromatic - $\mathrm{R}$ groups, with the NIT moiety possessing also oxidized and reduced forms that are stable at room temperature ${ }^{85}$ (Figure 1a,b). NIT$\mathrm{R}$ radicals contain a single spin centre delocalized over two N-O groups and the carbon atom in between, which also links to the peripheral - $\mathrm{R}$ functionality. They were chosen because they are interesting for organic switches and produced electronic devices with both $p$ and $n$ behaviours. ${ }^{87,88}$ Combined with tetrathiafulvalene moieties, they also showed conductive behaviour sensitive to the external magnetic field. ${ }^{89}$ The materials that we investigate here have a more insulating character than the tetrathiafulvalene-based compounds, and can thus be integrated into memristive and neuromorphic logic devices.

We can then tune the packing of the molecules by using different $-\mathrm{R}$ groups. In this way we obtain radicals with different levels of bulkiness and aromaticity, and all radicals investigated are shown in Figure 1b. This is particularly appealing to create electronic channels in the material, such as the one reported in Figure 1c for NIT-PhOMe.

All syntheses were performed as described in the supplementary material. ${ }^{90}$ Briefly, the NIT-R were prepared according to the published method by Ullman ${ }^{91,92}$ and, after purification via column chromatography and recrystallization, single crystals suitable for X-Ray diffraction were obtained. The structural data are reported in the supplementary material ${ }^{90}$ and show the presence of a variety of intermolecular channels, as useful for the purposes of the present investigation. No incorporation of solvents in the structure was ever observed.

The transport characteristics were measured using a 59 two-point-probe setup and were always repeated for sev- 60 eral crystals of each compound, so as to ensure the re- 61 producibility and check the variability among different 62 crystal qualities and batches (see Table III and S.I. ${ }^{90}{ }_{63}$ for details). The typical observed characteristics are ${ }_{64}$ reported in Figure 2a for crystals of all NIT-R com- 65 pound. All curves clearly show a linear and a non-linear 66 response at low and high voltages respectively. Both 67 regimes are roughly symmetrically placed around zero 68 voltage, with the residual asymmetries being attributable 69 to non-perfect contacts. The linear response observed at 70 low voltages in all curves can be assigned to ohmic be- 71 haviour, with measured resistances that fall in the tens of 72 $T \Omega$ range, about six orders of magnitude higher than in ${ }_{73}$ tetrathiafulvalene-based NIT-R derivatives. ${ }^{89}$ At higher ${ }_{74}$ voltages, in the non-linear region, resistances are in the 75 1-0.1 T $\Omega$ range, indicating the presence of different, less 76 conventional, transport channel.

The use of different sweep rates for the source-78 drain voltage leads to a hysteretic behaviour for the 79 fastest sweep rates, as often observed in organic so semiconductors ${ }^{93}$ that produce devices with high capac- 81 itances. Such effects can be minimized by decreasing the 8 s
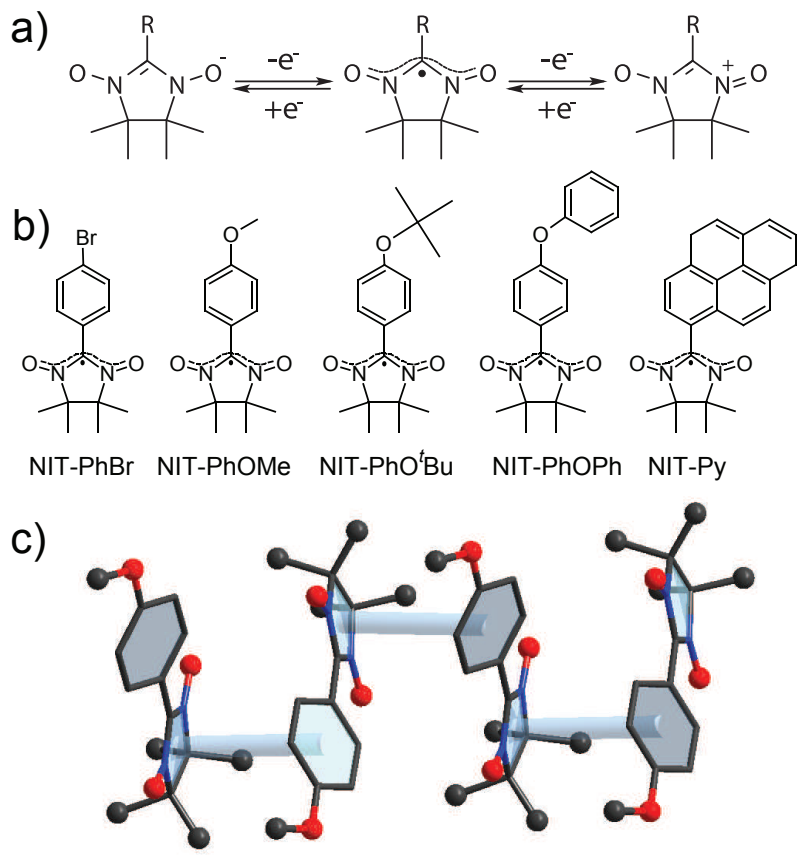

FIG. 1. (Color online) (a) Schematic representation of the general structure of the nitronyl-nitroxyde (NIT-R) radicals, showing the different oxidation states, available at room temperature, and the reduction/oxidation processes from the pristine neutral form. (b) Schematic representation of the five radicals investigated, with the corresponding acronyms below. (c) Crystal structure of radical NIT-PhOMe, with the cylinders highlighting the presence of overlap between the electronic clouds of aromatic appendages and the NIT moiety. Carbon is represented in black, oxygen in red, nitrogen in blue and hydrogen atoms are omitted for the sake of clarity.

sweep rate, as shown in Figure 2b, where we plot the dependence of the $I$ vs $V_{s d}$ curves on the voltage sweep rate of $V_{s d}$. In all the following measurements such capacitive effects were minimized by sweeping $V_{s d}$ slower than 0.2 $\mathrm{V} / \mathrm{s}$.

Very low energy sub- $\mathrm{THz}$ spectroscopy is a powerful tool to investigate the electronic properties of lowconducting materials, and affords an excellent way to extract the dielectric constant and information on the minimum mobility, $\mu_{\min }$ (see section III). In this technique, depicted in Figure 3a the surfaces of the pellets act as the mirrors of a Fabry-Perot resonator, so that interference effects produced by multiple reflections at the boundaries lead to an alternating transmission that depends on the wavelength, the sample thickness and the complex refractive index of the compound itself. ${ }^{94}$ In this low-energy range of the spectrum the real part of the optical dielectric constant, $\epsilon_{1}$, coincides with the DC relative permittivity of the material (i.e. $\epsilon_{r}$ in Equation 2 below) and valuable information on the conduction processes can be obtained. ${ }^{95,96}$ Assuming, as commonly done, that the frequency, $\omega$, dependence of the dielectric constants is negligible, we obtain the $\epsilon_{1}$ and $\epsilon_{2}$ values shown in Table I. 
TABLE I. Values of $\epsilon_{1}$ and $\epsilon_{2}$ as extracted from sub-THz measurements for the NIT-R radicals. $\epsilon_{1}$ values are obtained by averaging between 2 and $300 \mathrm{~K}$, while the $\epsilon_{2}$ ones refer to $250 \mathrm{~K}$ (see text).

\begin{tabular}{lcc}
\hline \hline NIT-R & $\epsilon_{1}$ & $\epsilon_{2}$ \\
\hline NIT-PhBr & $2.9 \pm 0.2$ & $6.7 \pm 0.2 \cdot 10^{-2}$ \\
NIT-PhOMe & $2.8 \pm 0.1$ & $2.9 \pm 0.2 \cdot 10^{-2}$ \\
NIT-PhOtBu & $2.79 \pm 0.06$ & $3.95 \pm 0.05 \cdot 10^{-2}$ \\
NIT-Py & $2.61 \pm 0.07$ & $8.18 \pm 0.05 \cdot 10^{-2}$ \\
\hline \hline
\end{tabular}

The transmission spectra for the analyzed compounds (Figure 3b), as obtained at a temperature of $10 \mathrm{~K}$ by dividing the spectrum of the pellet by that of the sources (acquired at exactly the same conditions), contain a superposition of two oscillations. The spectra are devoid of absorptions, except for the NIT-Py radical that shows a vibronic excitation at $20 \mathrm{~cm}^{-1}$. Contrarily to expectations for a Mott insulator, the overall transmission decreases with increasing wavenumber, owing to the nonvanishing value of $\epsilon_{2}$ (Table I). This is another hint for different transport channel within these compounds, and this aspect will be further discussed in Section IV. The different transmittivities observed are to be ascribed to different $\epsilon_{2}$ values and pellet thicknesses.

The temperature, $T$, and $\omega$ dependences of the response of the radicals always show two regions (see 3c for the behaviour of NIT-PhOMe, taken as a typical example): a low- $T$ region (from 5 to $50 \mathrm{~K}$ ), where no temperature evolution of the absorption is observed, and a $T>50 \mathrm{~K}$ region, where the transmission signal decreases with increasing $T$. We observe that $\epsilon_{2}$ is always relatively small, with a clear increase on increasing $T$ due to the higher out-of-phase response of thermally-generated free charge carriers. On the contrary, $\epsilon_{1}$ shows no T dependence within our experimental error and varies for the different NIT-R radicals between 2.6 and 3 (Table I). All NIT-R compounds show roughly the same $\epsilon_{1}$ value, often within our experimental error, and that this defines the range of values to be typically expected for such materials.

\section{EXTRACTION OF THE TRANSPORT PARAMETERS}

For each system we can define a threshold voltage $V_{c},{ }^{46}$ between the ohmic regime and non-linear one (Table II). ${ }^{47}$ Below $V_{c}$ the density of thermally-generated free carriers ${ }^{48}$ inside the crystal is dominant with respect to the injected 49 charge carriers. The behaviour is hence given by Ohm's ${ }^{50}$ law, which, neglecting diffusive contributions to the cur- ${ }^{51}$ rent, provides the current density:

$$
J_{\Omega}=\frac{I_{\Omega}}{W}=\mathrm{e}_{t h} \mu_{0} \frac{V_{\Omega}}{L}
$$
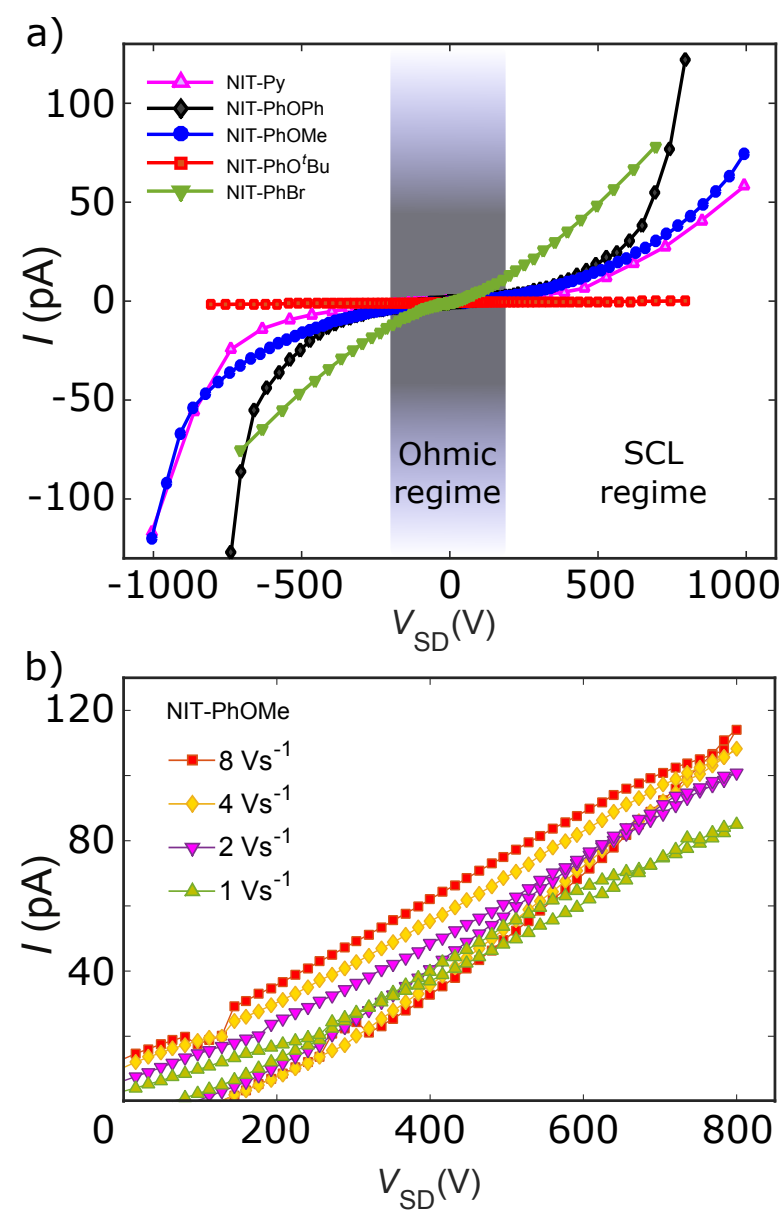

FIG. 2. (Color online) (a) Room temperature $I-V_{s d}$ characteristic curves resulting from averaging on multiple sets of measurements collected on NIT-PhBr (green down triangles), NIT-PhOMe (blue circles), NIT-PhOtBu (red squares), NIT$\mathrm{PhOPh}$ (black diamonds), and NIT-Py (violet up triangles). (b) Dependence of the $I$ vs $V_{s d}$ curves on the sweep rate of the bias voltage, as acquired for the NIT-PhOMe radical. Decreasing the sweep rate capacitive effects can be minimized as can be seen from the narrowing gap between forward and backward sweeps and decreased zero-voltage residual current.

$$
\text { the }
$$
tance $R_{S}=V_{\Omega} I_{\Omega}^{-1} W L^{-1}$ where we take $W=20 \mu \mathrm{m}$ as the tip diameter contacting the crystal and $L=400 \mu \mathrm{m}$ is the distance between the tips for each crystal. For all substituents - $\mathrm{R}$ attached to the NIT-R radical, we obtain high $R_{S}$ values (in the T $\Omega$ range, Table II) and this already provides important information on the transport mechanism present in the NIT-R family: the high sheet resistances indicate localized transport processes being dominant over delocalized ones. In order to corroborate 

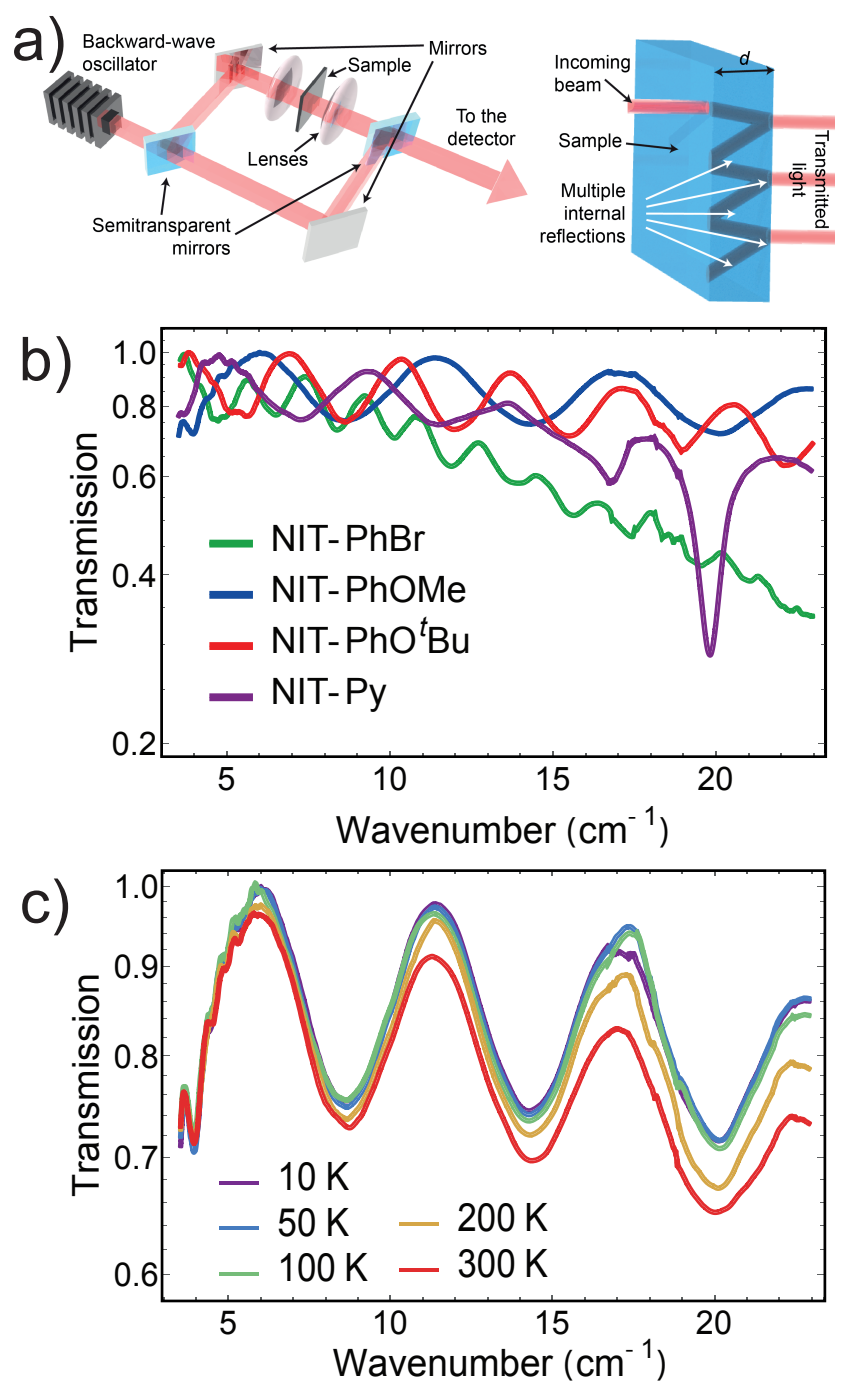

FIG. 3. (Color online) (a) Scheme of the $\mathrm{THz}$ spectrometer used for the measurements (left) and scheme of the multiple internal reflections within the sample, leading to FabryPerot interferences (right). (b) Transmission spectra in the $3-23 \mathrm{~cm}^{-1}$ range, as measured for the NIT-R compounds on pellets at $T=10 \mathrm{~K}$. (c) Temperature and frequency dependence of the transmission spectra of NIT-PhOMe in the $3^{-}{ }^{16}$ $23 \mathrm{~cm}^{-1}$ and $10-300 \mathrm{~K}$ ranges. A vertical logarithmic scale is ${ }^{17}$ used for clarity. this observation, the carrier mobility, which can be de- ${ }^{21}$ rived from the measurements as shown below, is a useful ${ }_{23}^{22}$ parameter to start distinguishing between the different ${ }_{24}^{23}$ possible transport mechanisms. The boundary between ${ }_{25}$ delocalized and localized transport processes, i.e. between band and hopping transport is commonly consid- ${ }_{27}$ ered at charge carrier mobilities in the range of $0.1-1$ $\mathrm{cm}^{2} V^{-1} s^{-1} .^{81,84}$ For all NIT-Rs, extremely low mobil- ${ }_{29}^{28}$ ities in the order of $10^{-3}$ to $10^{-6} \mathrm{~cm}^{2} V^{-1} s^{-1}$ are ob- ${ }_{30}$ tained, indicating that inter-molecular hopping transport ${ }_{31}$ is dominant (Figure 1a).

The non-linear behaviour at high voltages is more in- 33
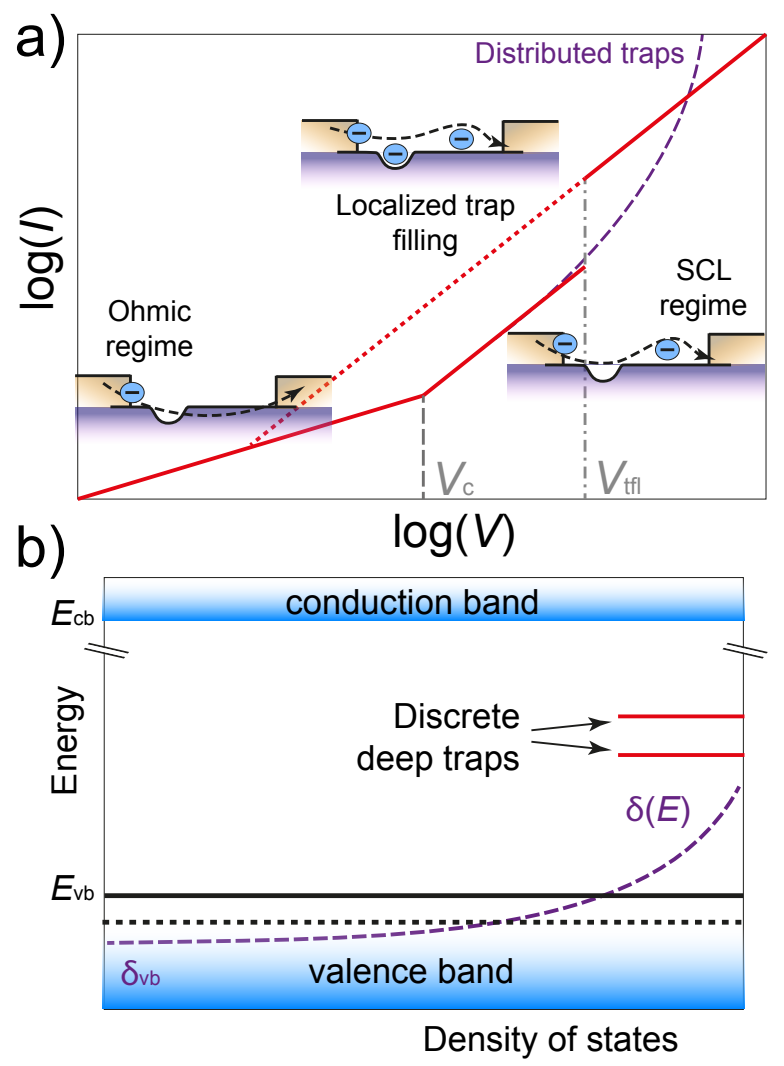

FIG. 4. (Color online) (a) Schematic representation of the different conduction regimes available for the NIT-R radicals. The ohmic regime, present at low voltages, gives way, at higher voltages, to surface charge limited transport with possible trap effects. The effects of exponentially-distributed (violet) and localized-energy (red) traps are also shown (b) Schematic depiction of the different types of traps available for NIT-R systems, with the localized traps (red lines) and energetically-distributed traps (violet curve) placed between the valence and conduction bands of the system.

triguing: insulating crystals, ${ }^{97}$ organic semiconductors (both in crystalline ${ }^{98}$ and film ${ }^{81,83}$ forms) and even semiconducting nanowires ${ }^{99-101}$ may display complex transport phenomena such as space-charge limited (SCL) currents. ${ }^{102}$ SCL transport arises when the contacts to the electron reservoirs are ohmic and the material is not a good conductor, so that at high voltages an excess of free carriers is injected into the material in the vicinity of the contact. In this situation, the current through the material is no longer limited by its resistance, but by the accumulated space charge, which clogs the conductance channel. As a result, SCL currents allow the investigation of many intrinsic properties of the material that are not easily available in the standard ohmic regime, such as charge carrier mobility, thermal-equilibrium carrier concentration and charge trap densities (Figure 4). For NIT-Rs, due to both the high resistance of the bulk material and the contact geometry, the systems will follow surface SCL currents, rather than bulk SCL. ${ }^{77}$ The 
main characteristic of SCL currents is the presence of a non-injection-limited regime after the ohmic regime, i.e. above the crossover voltage $V_{c}$, where $I$ is proportional to $A V^{2}$, with a proportionality constant $A$ that depends on the contact geometry. In our case, where two electric probing tips contact the molecular crystal at the same crystal surface, the relationship for surface SCL currents is: ${ }^{77}$

$$
I_{S C L}=\frac{2}{\pi} \frac{\mu_{\min } \epsilon_{0} \epsilon_{r} W}{L^{2}} V^{2}
$$

where $\epsilon_{r}$ is the dielectric constant of the material and $\mu_{\min }$ is the minimum carrier mobility, which includes the contact quality and the presence of traps in the electron channel.

Variations in the contact and channel characteristics can influence the absolute current values in this regime, generating sensible crystal-to-crystal variability. Small curve asymmetries for positive and negative voltages are also consistent with these effects, and are usually ascribed to different hole and electron trapping potentials. This variability can be taken in account by performing a statistical analysis on the slope of the $I$ versus $V$ curves, which is related to the conduction mechanisms and is independent on the absolute value of the resistivity. A common way to identify the presence of SCL currents is by plotting the linear conductance $G=I / V$ vs $V$ in a log-log plot, which will yield a straight horizontal line for the ohmic regime, and a sudden increase in conductivity once the SCL regime triggers. All NIT-Rs show this behaviour (Figure $5 \mathrm{a}$ ), whatever $R$, clearly highlighting the presence of SCL currents.

We can now combine the optical and transport measurements to obtain information on the minimum mobility $\left(\mu_{\text {min }}\right)$ as, at the $\omega$ used, the extracted $\epsilon_{1}$ coincides with $\epsilon_{r}$ in Equation 2. It should be noticed that all NIT-R show roughly the same $\epsilon_{1}$ value, and that variations much larger than those observed in the series do not introduce important variations in the extracted $\mu_{\text {min }}$. Thus the $\epsilon_{1}$ of NIT-PhOPh can be safely considered to be, for our purposes, $2.8 \pm 0.2$. We can thus extract $\mu_{\text {min }}$ for all compounds, using $W=20 \mu \mathrm{m}$ and $L=400 \mu \mathrm{m}$ as before (Table II) and can be seen that strongly depends on the radical substituent, with NIT-PhBr exhibiting the highest one, followed by NIT-PhOMe and NIT-PhOPh. Eventually the lowest mobilities belong to NIT-PhOtBu and NIT-Py, which show very similar conductivities, at the limit of our detection range. Information on the conduction mechanisms can be extracted from the voltage threshold, Vc, between the ohmic and SCL regimes, where we have $I_{\Omega}=I_{S C L}$ and thus we can evaluate the thermal-equilibrium carrier density at the crystal surface by:

$$
n_{t h}=\frac{2}{\pi} \frac{\epsilon_{0} \epsilon_{r}}{\mathrm{e}} \frac{V_{c}}{L}
$$

To extract the threshold voltages we can plot the cur- 58 rent in both the ohmic and the SCL regimes in a double 59
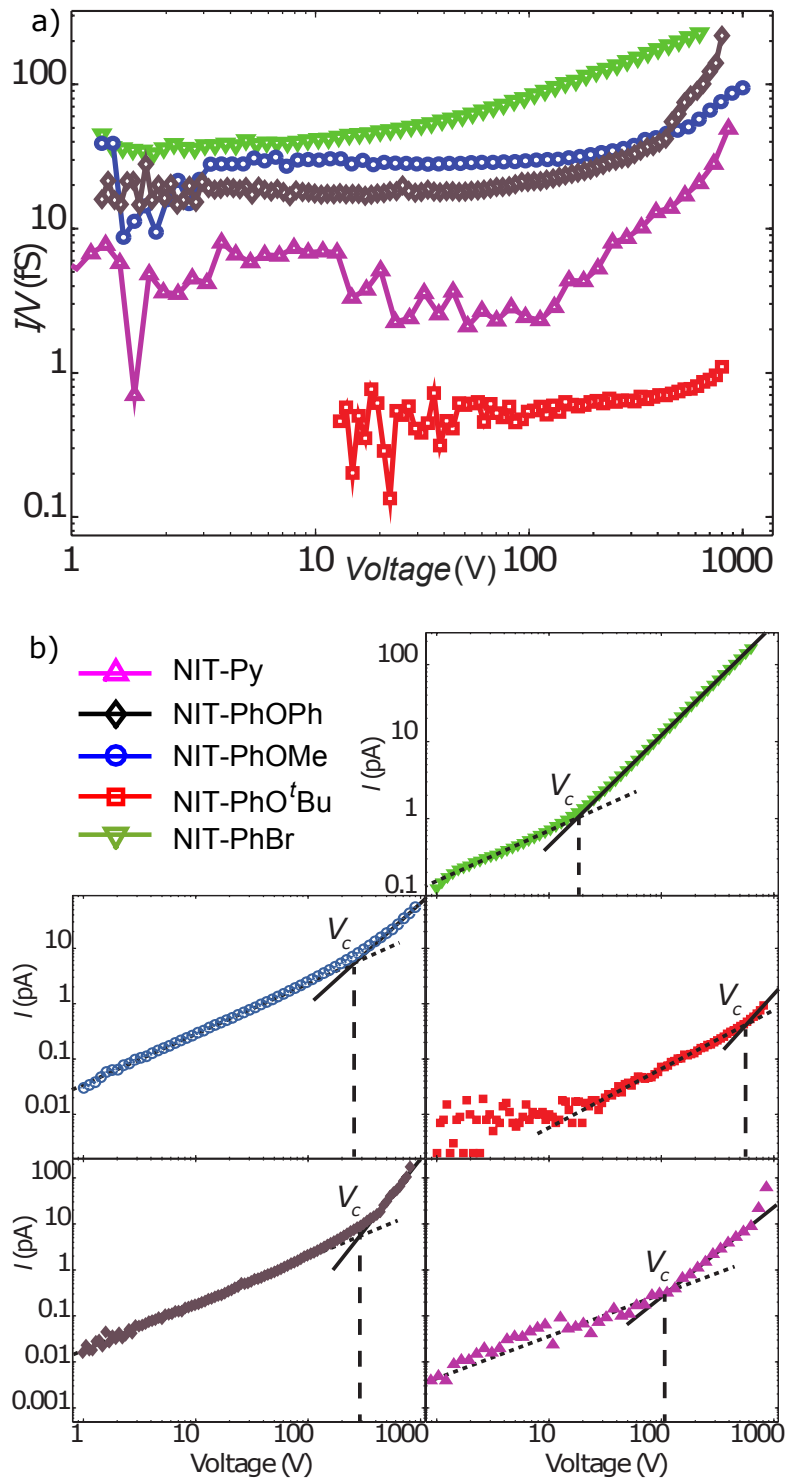

FIG. 5. (Color online) (a) Bi-logarithmic plot of the voltage dependence of the conductance for all NIT-R compounds, showing the constant value for lower voltages, connected to the ohmic regime, and the raising in conductance at higher voltages, where SCL effects become dominant. (b) Bi-logarithmic plots of the current vs source-drain voltage characteristic curves for the different NIT-R compounds. The dashed and continuous lines show the fitting for the Ohmic and SCL regimes, respectively (see Table II and Figure 6 for the results). The crossover voltages $V_{c}$, as obtained from the intersection of the two regimes, are also highlighted with vertical lines.

logarithmic plot (Figure 5b-f) and then fit the curves with the function $I=G_{i} V^{\eta}$, where the $i$ index stands for the ohmic $(i=\Omega)$ or charge-limited $(i=\mathrm{SCL})$ regions and the exponent $\eta$ varies between the two regimes. $V_{c}$ is then obtained from the intercept between the fits of the high and low voltage regions. In order to account for the variability among the different sample geometries and 
TABLE II. Minimum mobility $\mu_{m i n}$, surface thermal carrier density $n_{t h}$, sheet resistance $R_{S}$, crossover voltage $V_{c}$, Ohmic conductance and fitting exponent $\left(G_{\Omega}\right.$ and $\left.\eta_{\Omega}\right)$, charge-limited parameters $G_{S C L}$ and $\eta_{S C L}$ and low-field mobility $\mu_{0}$ for the different NIT-R radicals, as extracted from the optical and transport measurements.

\begin{tabular}{lccccccccc}
\hline \hline NIT-R & $\begin{array}{c}\mu_{\min } \\
{\left[\mathrm{cm}^{2} \mathrm{~V}^{-1} \mathrm{~s}^{-1}\right]}\end{array}$ & $\begin{array}{c}n_{t h}(2 \mathrm{D}) \\
{\left[10^{13} \mathrm{~m}^{-2}\right]}\end{array}$ & $\begin{array}{c}R_{S} \\
{[\mathrm{~T} \Omega]}\end{array}$ & $\begin{array}{c}V_{c}^{\mathrm{a}} \\
{[\mathrm{V}]}\end{array}$ & $\begin{array}{c}G_{\Omega}{ }^{\mathrm{a}} \\
{[\mathrm{fS}]}\end{array}$ & $\eta_{\Omega}{ }^{\mathrm{a}}$ & $\begin{array}{c}G_{S C L}{ }^{\mathrm{a}} \\
{\left[\mathrm{fSV}^{\eta-1}\right]}\end{array}$ & $\begin{array}{c}\eta_{S C L}{ }^{\mathrm{a}} \\
{\left[\mathrm{cm}^{2} \mathrm{~V}^{-1} \mathrm{~s}^{-1}\right]}\end{array}$ \\
\hline NIT-PhBr & $3.0 \pm 0.5 \cdot 10^{-2}$ & $1.0 \pm 0.5$ & $0.9 \pm 0.3$ & $40 \pm 20$ & $55 \pm 10$ & $0.9 \pm 0.3$ & $6.3 \pm 0.9$ & $1.5 \pm 0.1$ & $6 \pm 4 \cdot 10^{-3}$ \\
NIT-PhOMe & $4 \pm 3 \cdot 10^{-3}$ & $4.2 \pm 0.8$ & $2.2 \pm 0.3$ & $170 \pm 30$ & $23 \pm 3$ & $1.2 \pm 0.1$ & $0.9 \pm 0.6$ & $1.7 \pm 0.1$ & $7 \pm 1 \cdot 10^{-4}$ \\
NIT-PhO ${ }^{t} \mathrm{Bu}$ & $7 \pm 4 \cdot 10^{-4}$ & $17 \pm 2$ & $75 \pm 4$ & $510 \pm 40$ & $0.89 \pm 0.03$ & $0.9 \pm 0.3$ & $0.2 \pm 0.1$ & $1.5 \pm 0.3$ & $5.0 \pm 0.5 \cdot 10^{-6}$ \\
NIT-PhOPh & $6 \pm 3 \cdot 10^{-3}$ & $3 \pm 1$ & $3 \pm 1$ & $130 \pm 40$ & $18 \pm 8$ & $1.1 \pm 0.2$ & $1.2 \pm 0.7$ & $2.0 \pm 0.3$ & $7 \pm 4 \cdot 10^{-4}$ \\
NIT-Py & $1.4 \pm 0.9 \cdot 10^{-4}$ & $0.9 \pm 0.4$ & $5 \pm 3$ & $60 \pm 30$ & $6 \pm 3$ & $0.9 \pm 0.5$ & $1 \pm 0.9 \cdot 10^{-2}$ & $2.1 \pm 0.3$ & $1.4 \pm 0.9 \cdot 10^{-3}$ \\
\hline \hline
\end{tabular}

${ }^{\text {a }}$ Errors represent the widths of the statistical distributions

b Values refer to $\epsilon_{1}=2.8 \pm 0.2$, see text

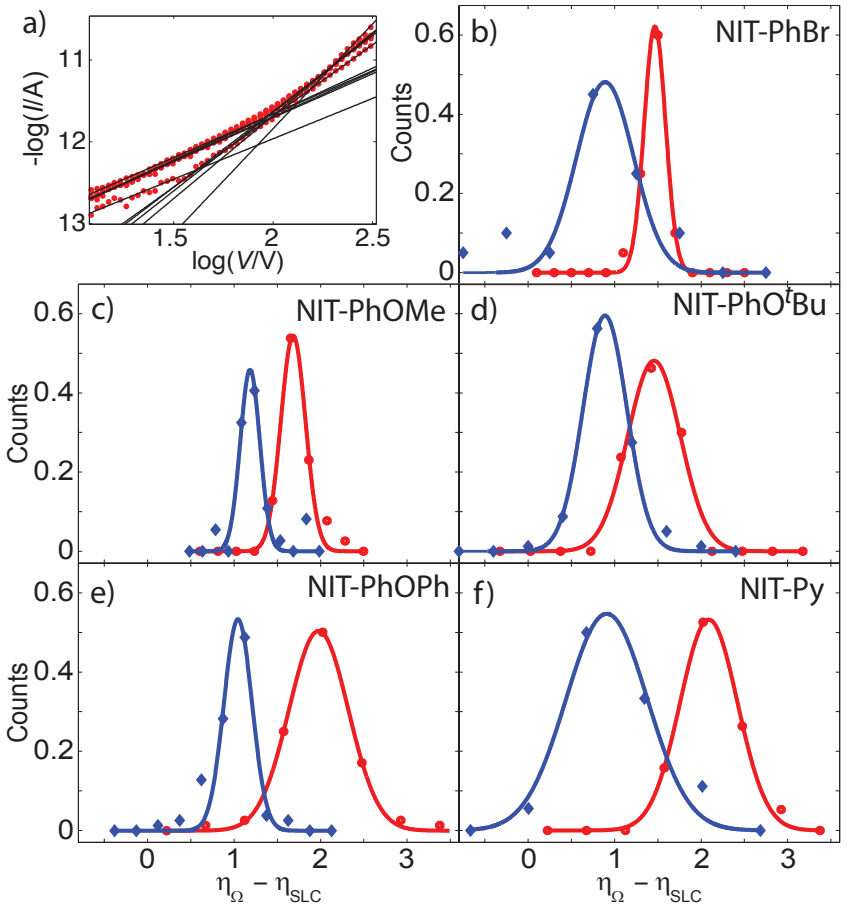

FIG. 6. (Color online) (a) Example of fitting of the different ${ }_{17}$ regimes for a number of measurements performed on NIT- ${ }_{18}$ PhOMe, allowing the statistical analysis of the conduction ${ }_{19}$ regimes. Details about data sets in Table III. The resulting distribution of the exponents, as obtained for the low- ${ }^{20}$ voltage (blue diamonds) and high-voltage (red dots) regimes ${ }^{21}$ are shown in: b) for NIT-PhBr; c) for NIT-PhOMe; d) for ${ }^{22}$ $\mathrm{NIT}-\mathrm{PhOtBu}$; e) for NIT-PhOPh; f) for NIT-Py. No-count ${ }^{23}$ points are shown for clarity and solid lines correspond to gaus- 24 sian fits (values in Table II).

25 measurements, a statistical analysis was performed by acquiring several sets of measurements for each compound 26 on different crystals (see supplement information ${ }^{90}$ ), and ${ }_{27}$ performing the fitting procedures on all of them (Figure 28 6a shows a small subset of measurements). The result- 29 ing statistical analysis provided a gaussian distribution 30 for each exponent, as shown in Figure 6, from which the 31
TABLE III. Specifications on the number of measurements used for statistical analysis. Several I-V cycles were performed on the indicated number of crystals. It should be noticed that each curve contributed four points to our analysis, for forward and backward sweeps at positive and negative voltages.

\begin{tabular}{lcc}
\hline \hline NIT-R & Total I-V curves & Number of Crystals \\
\hline NIT-PhBr & 15 & 2 \\
NIT-PhOMe & 23 & 3 \\
NIT-PhO $^{t} \mathrm{Bu}$ & 27 & 2 \\
NIT-PhOPh & 45 & 3 \\
NIT-Py & 5 & 1 \\
\hline \hline
\end{tabular}

values provided in Table II were extracted. All compounds with the higher mobilities show a value of the SCL exponent slightly below the predicted value of 2 , while the two systems with the lowest conductance and the least efficient intermolecular channels provide exactly 2 , within experimental error. These compounds also display a wider dispersion of behaviours, with a few cases showing exponents up to 3 . This effect is actually to be linked to a more limited experimental accuracy, produced by the presence of other mechanisms, such as traps (see Section V) and the limited voltage range between $V_{C}$ and the breakdown voltage of the crystals. All compounds show an effective thermal carrier density in the order of $10^{13} \mathrm{~m}^{-2}$ at the crystal surface, with only a small dependence on the - $\mathrm{R}$ group observable, in perfect compatibility with the expected variability in the quality of the crystal-electrodes contacts.

\section{MODELING OF THE TRANSPORT}

In a pure Mott insulator the main parameter that influences the conductance is the charging energy needed to add an electron to a neutral molecule. For the NIT$\mathrm{R}$ systems the main parameter are the distance between the upper and lower Hubbard bands and the energy distances of the singly-occupied molecular orbital (SOMO 


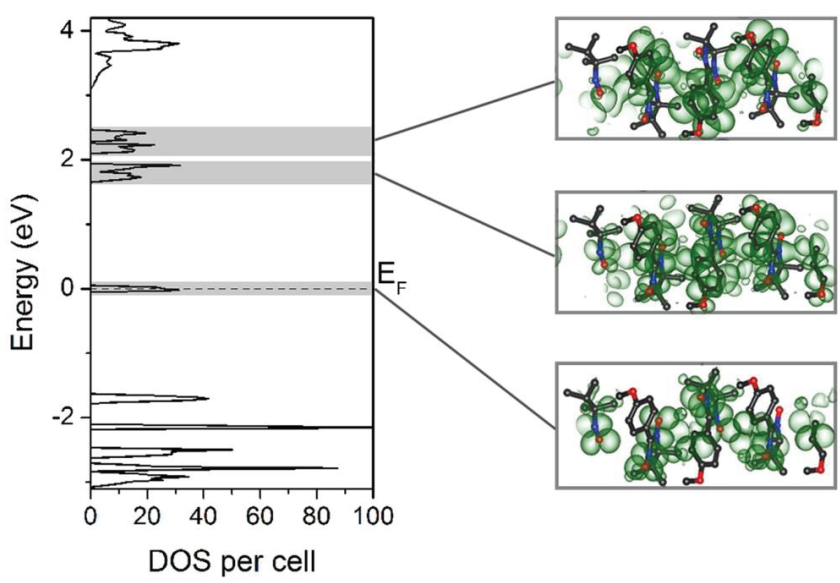

35

FIG. 7. (Color Online) Left: Calculated DOS for NIT-PhOMe ${ }^{36}$ showing the different densities close to $E_{F}$ and charge den- 37 sity maps obtained by projecting in real space the states in 38 different energy intervals, as highlighted by the shaded areas. 39 Right: Charge densitity maps obtained for NIT-PhOMe by ${ }_{40}$ projecting in real space the states around $E_{F}$ (bottom panel) ${ }_{41}$ and around $2 \mathrm{eV}$ (upper panels). or $\alpha$-HOMO) and the lowest unoccupied molecular or- ${ }_{45}$ bital (LUMO) from the highest occupied molecular or- 46 bital (HOMO). ${ }^{103}$ To gain insight into these parameters, ${ }_{47}$ we performed numerical calculations with full geome- 48 try optimizations of the NIT-Rs carried out using the ${ }_{49}$ density functional theory method (DFT) at the B3LYP ${ }_{50}$ level. ${ }^{104}$ All atoms were assigned a $6-311++\mathrm{G}^{* *}$ basis ${ }_{51}$ set and calculations were performed with Spartan14 pro- ${ }_{52}$ gram packages. ${ }^{105}$ The results (Table IV) show that the ${ }_{53}$ energy differences between the three orbitals are identi- ${ }_{54}$ cal for all compounds except for NIT-Py, which is only ${ }_{55}$ a little higher. This is in good agreement with our es- ${ }_{56}^{56}$ timation of the number of carriers $n_{t h}$ (Table II) but is ${ }_{57}^{56}$ not enough to explain the large conductivity differences, ${ }_{58}$ indicating that the main limiting factor is not the molec- ${ }_{50}$ ular charging energy itself, but the intermolecular barrier ${ }_{60}$ that has to be overcome for the transport process to occur. As such barriers are much more sensitive to the ${ }_{62}^{61}$ molecular arrangement in the bulk and the steric of the ${ }_{63}$ -R substituents can thus become an important factor.

Insight on this intermolecular conduction path can ${ }_{65}$ be obtained theoretically by DFT calculations on the 60 crystal structure of the NIT-PhOMe system, which has ${ }_{67}$ a smaller unit cell compared to the others, contain- ${ }_{68}$ ing only four molecular units, and can thus be treated ${ }_{69}$ with reasonable computing power. For these electronic ${ }_{70}$ structure calculations we employed the Vienna ab ini- ${ }_{71}$ tio simulation package (VASP) ${ }^{106-109}$ using the projec- ${ }_{72}$ tor augmented-wave (PAW) pseudopotentials ${ }^{110}$ and the ${ }_{73}$ Perdew-Burke-Ernzerhof generalized gradient approxi- ${ }_{74}$ mation (PBE-GGA). ${ }^{111}$

The energy cutoff for the plane wave basis set was put 76 at $400 \mathrm{eV}$ and the first Brillouin zone was sampled with 77 a $7 x 7 x 7$ Monkhorst-Pack k-points mesh. ${ }^{112}$ The resulting 78
TABLE IV. Calculated energy distances of the SOMO and LUMO orbitals from the HOMO, crystallographic distances and tilting angles between the aromatic components of adjacent molecules, for the different NIT-R compounds.

\begin{tabular}{lcccc}
\hline \hline NIT-R & SOMO $[\mathrm{eV}]$ & LUMO $[\mathrm{eV}]$ & $\mathrm{r}[\AA]$ & angle $\left[{ }^{\circ}\right]$ \\
\hline NIT-PhBr & 0.7 & 3.6 & 4.760 & 4.08 \\
NIT-PhOMe & 0.4 & 3.3 & 4.332 & 33.23 \\
NIT-PhO $^{t} \mathrm{Bu}$ & 0.6 & 3.5 & 5.911 & 56.15 \\
NIT-PhOPh & 0.5 & 3.4 & n.a. & n.a. \\
NIT-Py & 0.3 & 3.3 & 5.895 & 43.13 \\
\hline \hline
\end{tabular}

orbitals, as depicted in real space (figure 7, right), show a fragmented structure, with no clear conduction path. On the contrary the orbitals above the Fermi energy show a delocalization of the states. Analysis as a function of the strength of the on-site interaction $U$ in a DFT $+U$ approach revealed that no bandgap is opened at the Fermi level even for $U$ up to $6 \mathrm{eV}$. Such levels of interaction did not qualitatively change the density of states of the radicals at all, indicating that the charge transport does not follow the standard mechanisms of a Mott insulator. On the contrary, analysis of the charge density maps, as obtained by projecting in real space all states belonging to specific energy intervals shows the localized characteristic of the states around $E_{F}$ and the delocalized conduction channels of the states between 1.5 and $2.5 \mathrm{eV}$. These numerical results thus support the idea that NIT-Rs do not completely behave as Mott insulators: instead they sport a minor density of states around the Fermi energy, which can be easily filled, so that conduction electrons must then be injected at much higher energies.

We can experimentally verify this hypothesis by varying the intermolecular channel via the different -R groups in the systems. $\pi$-stacking interactions between each aromatic phenyl group of the -R appendages and the NIT group of the neighbouring molecule, as depicted in Figure 1c for NIT-PhOMe, is found in all compounds, but with very considerable differences. Radicals with bulky -R substituents that hinder intermolecular interactions, lead to intermolecular distances along the channel and tilting angles (taken between the planes of the phenyl ring and of the five-membered heterocycle of the NIT group) that deviate considerably from the standard $\pi$ stacking values (Table IV). We can observe that systems with the larger distances between the stacking aromatic groups display indeed lower conductances. The effect is particularly clearcut in NIT-PhOtBu and NIT-Py, where the large tilt between the two planes produces a disruption of the stacking pattern and extremely low conductances. These structural trends confirm that the conductance is not simply limited by a Mott mechanism, but is assisted by a developed intermolecular electron channel. By applying high voltages we can thus inject electrons into the material which can diffuse through it exploiting these overlapping channels. It should be noticed that the 


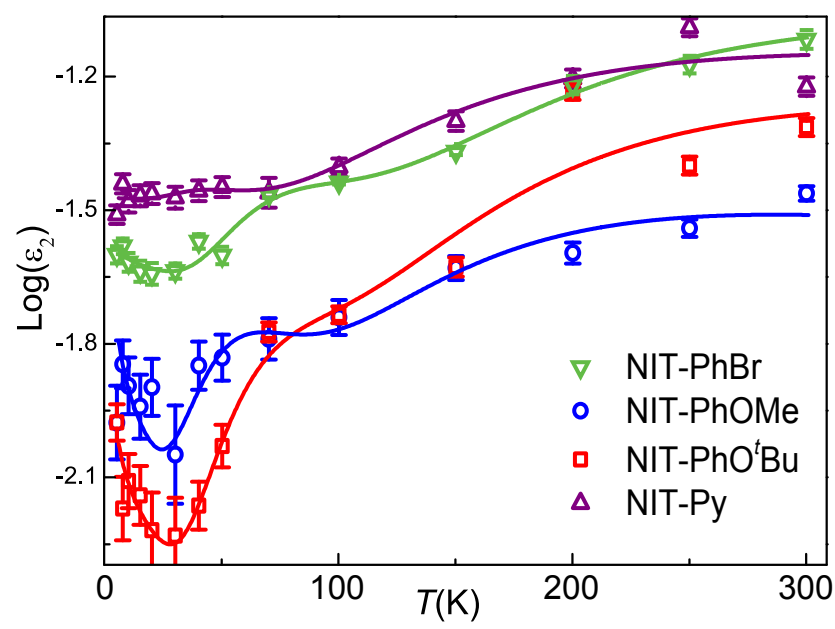

FIG. 8. (Color online) Temperature dependence of the logarithm of the optical $\epsilon_{2}$ value, for NIT-PhBr (green triangles), NIT-PhOMe (blue dots), NIT-PhOtBu (red squares) and NIT-Py (violet triangles), as determined from the fitting of the sub-THz spectra. Solid lines correspond to fits with the expected behavior for small polarons and low-temperature residual conductivity, as described in the text.

orbital overlaps we consider here are between neighbouring molecules, and are not intramolecular ones (Figure 7, right).

These conclusions are also corroborated by the $T$ de- ${ }^{32}$ pendence of $\epsilon_{2}$, whose logarithm is reported in Fig- ${ }^{33}$ ure 8. The optical $\epsilon_{2}$, at low photon frequencies $\omega,{ }^{34}$ is directly related to the mobility of the carriers $\mu$ by ${ }^{35}$ the relation $\epsilon_{2}=A \mu$ (with the proportionality constant ${ }^{94} 36$ $\left.A=\omega /\left(4 e \pi n_{t h}\right)\right)$. The data do not scale as expected for ${ }^{37}$ Mott-insulators $^{90}$, and can only be reproduced, for all ${ }^{38}$ NIT-R, by considering hopping of small polarons ${ }^{113}$ in the Holstein model. The low- $T$ minimum, clearly present for all NIT-R, can be understood in the framework of transport theories of electron hopping among molecules assisted by vibrational effects modulating the electronic ${ }^{39}$ channel. ${ }^{84,113,114}$ According to these models the electron ${ }^{40}$ mobility should first decrease, on lowering $T$, because of ${ }^{41}$ the progressive decrease of available vibrational modes ${ }^{42}$ that assist the hopping, as indeed observed (Figure 8) ${ }^{43}$ down to $T \approx 30 \mathrm{~K}$. The analytical form for the mobility: ${ }^{44}$

$$
\mu_{\text {pol }}=\frac{\pi^{1 / 2} e a^{2} J^{2} \mathrm{e}^{-\frac{W_{p}}{2 \mathrm{k}_{B} T}}}{\hbar\left(2 W_{p}\right)^{1 / 2}\left(\mathrm{k}_{B} T\right)^{3 / 2}}
$$

relies on the lattice constant a of the crystal in which 49 the small polaron occurs, the polaron interaction poten- 50 tial $J$ and the thermal activation energy for hopping $W_{p}$, given by half the small-polaron binding energy. All the extracted polaron energies are in the order of $0.4 \mathrm{eV},{ }^{51}$ about the magnitude of the HOMO-SOMO distance, and comparable to the energies expected from the DFT cal- ${ }_{52}$ culations to reach the delocalized electron channel, con- 53 firming, again, the relevance of this non Mott-like inter- 54 molecular channel.
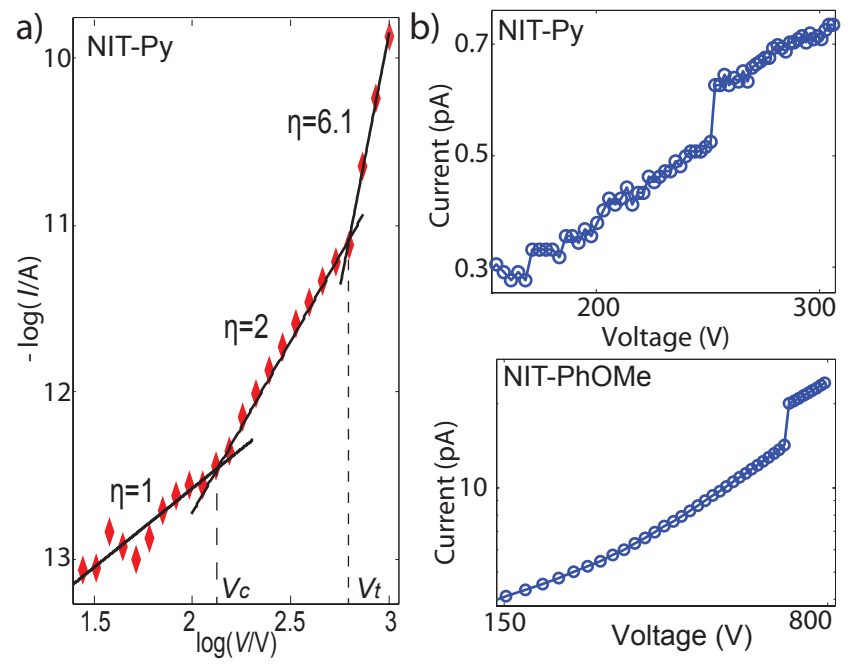

FIG. 9. (Color online) (a) Example of the presence of distributed traps within the SOMO-LUMO gap, as observed on a bilogaritmic plot. The black lines represent the exponents that indicate the conductivity regime of each part of the curve. Over $V_{t}=500 \mathrm{~V}$ the effect of distributed traps is clearly visible as explained in the text. (b) Examples of localized traps for NIT-Py (top) and NIT-PhOMe (bottom).

Below $30 \mathrm{~K}$ an increase of $\epsilon_{2}$ is typically observed. This cannot be accounted by simple hopping, and such observations are typically ascribed to the effects of polaron tunneling and residual band-like conductivity, again in agreement with the aforementioned DFT calculations. To account for these effects we can add two terms to Equation 4, accounting for the tunneling and the bandlike conductance:

$$
\mu_{L T}=\mu_{t u n}+\mu_{B L}=\frac{e a^{2}}{\hbar \gamma k_{B} T}\left[\frac{\gamma}{\pi} \xi\right]^{\frac{1}{2}} \mathrm{e}^{(-2 \gamma \xi)}+d T^{-n}
$$

where $\xi=\operatorname{csch}\left(\frac{W_{p}}{2 \gamma k_{B} T}\right), \gamma$ is a measure the strength of the electron phonon coupling and $n$ is an empirical exponential describing the band-like processes. The expression reproduces well all curves for all NIT-R radicals down to $2 \mathrm{~K}$, and the $\gamma$ term is always found to be between 2 and 3 , which still indicates a relatively weak electron-phonon coupling, even though higher than many organic semiconductors. In all cases we could not reproduce the data with the tunneling term $\mu_{\text {tun }}$ alone, without including the power-law dependence of $\mu_{B L}$. This confirms again the very important role of the intermolecular interaction channels.

\section{TRAP EFFECTS}

Trap states and additional conduction paths can become relevant at higher voltages. ${ }^{115,116}$ Figure 9 shows examples of the very appreciable deviations from the purely quadratic $I$ vs $V$ dependence that can be ob- 
served. Both experiments ${ }^{81}$ and theory ${ }^{117,118}$ indicate ${ }_{52}$ that surface traps can strongly influence the SCL regime ${ }_{53}$ of molecular crystals, with traps often created at the ${ }_{54}$ crystal-contact interface and at defective molecular sites. 55 In the present case we can identify two main trapping 56 mechanism (Figure 9). The first type of traps pro- 57 duces an exponential distribution of trap states below the 58 LUMO, described by ${ }^{119} \rho_{t}(E)=\left(\delta_{t} / E_{t}\right) \exp \left(E / E_{t}\right)$ where 59 $\delta_{t}$ is the trap density, $E_{t}$ the characteristic trap depth 60 and $E$ the energy relative to the conduction or valence band. These traps are gradually filled, leading to a current that increases faster than quadratic following the expression: ${ }^{119}$

$$
I \propto V^{m+1} / d^{2 m+1}
$$

where $m$ is given by $m=E_{t} / k_{B} T>1$ and $d$ is the thick- ${ }^{64}$ ness of the sample. As the resulting current only depends on the trap depth, but is independent of the device ge- ${ }^{60}$ ometry, a power law dependence of the $I$ vs $V$ charac- ${ }^{-}$ teristics with such a behaviour can be regarded as indi- ${ }^{60}$ cation for trap-limited SCL processes. Exponentially- ${ }^{60}$ distributed traps can explain the deviations from the ${ }_{71}^{70}$ quadratic $I$ vs $V$ behaviour wherever $\eta>2$ is observed, as, for example, in NIT-Py in Figure 9. Anyway, the ${ }_{73}$ NIT-R systems show far better agreement with the ideal SCL regime than most other molecular conductors, possibly due to the low energy of the traps and the surface SCL mechanism: for example in $\mathrm{AlQ}_{3}$, values of $6<m<10$ have been found, with trap energies $E_{t}=0.15$ $\mathrm{eV} \cdot{ }^{120,121}$ As the present theoretical background for dis- ${ }^{75}$ tributed traps considers neither hopping transport nor ${ }^{76}$ surface conductance, ${ }^{122}$ the behaviour is best modeled by ${ }^{77}$ introducing a field-dependent mobility, without consider- ${ }^{78}$ ing a theoretically-derived distribution of traps and char- ${ }^{79}$ acteristic temperatures, as already employed for $\mathrm{AlQ}_{3}{ }^{80}$ and PPV devices. ${ }^{123,124}$ We can then assume the mobil- ${ }^{81}$ ity to depend on the electric field E via the Poole-Frenkel ${ }^{82}$ mechanism: ${ }^{117}$

$$
\mu(E)=\mu_{0} \exp \left(\frac{\mathrm{e}^{3 / 2}}{\mathrm{k}_{\mathrm{B}} T} \sqrt{\frac{E}{\pi \epsilon_{0} \epsilon_{r}}}\right)
$$

where $\mu_{0}$ is the low-field mobility ${ }^{81}$ With the previously- 89 extracted thermal charge carrier density we can so cal- 90 culate $\mu_{0}$ with Equation 1 and substitute $\mu_{m}$ in by the ${ }_{91}$ Poole-Frenkel mobility $\mu(E)$ in Equation 7 . The result- 92 ing $I$ vs $V$ dependence can be used to describe the super- 93 quadratic behaviour at higher voltages that some of the 94 compounds show (see Figure 9a). Higher distributed trap 95 densities in the proximity of the electrodes are likely re- 96 sponsible for the underestimated currents close to Vc and ${ }_{97}$ any remaining sweep rate-independent discrepancies be- 98 tween the forward and backward sweeps (Figure 2).

The second kind of traps that can be observed are lo-100 calized electron traps, energetically located deep in the 101 band-gap of the material. ${ }^{81}$ In NIT-R systems these traps102 can take the form of locally-altered molecules, oxidized ${ }_{103}$ or reduced single molecules or chemically-defective regions, which can arise, for example, by the presence of the amino-nitroxide side product radical in the crystalline NIT-R. Two examples of such traps are shown in Figure $9 \mathrm{~b}$, with clear signatures of vertical jumps at the so-called trap-filling voltage $V_{t f l}$, above which transport is in the so-called trap-filled limit and continues to follow the quadratic voltage dependence. In the case of surface SCL currents, traps are filled by the injected charge at:

$$
V_{T F L}=\frac{\pi}{4 \epsilon_{0} \epsilon_{r}} \mathrm{e} L \delta_{d}
$$

from which the trap density per unit surface area, $\delta_{d}$ can be evaluated (the subscript $d$ denotes deep-traps, located at several times $k_{B} T$ from the valence band). This is also visible from the current jumps due to trap filling, which would be absent for a dense distribution of traps close to the conduction band. The presence of such deep traps, which are typically filled close to $0.4 \mathrm{eV}$, is typical of systems with very wide band-gaps, and would match with a gap width close to the $3 \mathrm{eV}$ as previously discussed. The observed densities of localized traps are ca. $\delta_{d}=3.5 \pm 0.3 \cdot 10^{10} \mathrm{~cm}^{2}$, which agrees with known densities in organic conductors ${ }^{125-129}$ and, in particular, to those of rubrene crystals. ${ }^{130}$

\section{CONCLUSIONS}

In conclusion we have shown that, contrarily to what conjectured up to now, the widespread compounds of the NIT-R class do not behave as typical Mott insulators when $-\mathrm{R}$ is aromatic, owing to interactions between the $\pi$ electronic cloud of the phenyl appendix and the delocalized NIT motif. These results are fundamental for the creation of spintronic nanodevices based on NIT-R radicals and allow using chemical design to tune the conduction properties of these materials. The spacecharge limited currents and strong trap effects here evidenced for the first time will be fundamental for the design of any nanoscale devices based on such materials, and in particular for latch memory, field-effect and spintronic devices. ${ }^{131-138}$ The observed mobilities fall in the correct range for the creation of memristive devices, where charge-limiting effects can be desirable to create nanometer-scale logic units. Such effects, which have until now been ignored, will thus be of fundamental importance for proposed molecular switching devices ${ }^{13}$ at the nanoscale.

In this paper we have also set a methodological standard for the investigation of the conduction regimes of low-conductive magnetic compounds, ${ }^{96}$ by providing a unique combination of optical measurements at sub- $\mathrm{THz}$ frequencies, transport data and numerical calculations. The results will constitute the experimental and theoretical backbone for the study of electron transport in NIT-R-based complexes, ${ }^{96}$ which are attracting particular interest for their slow-relaxing behaviour ${ }^{69}$ 
and the possibility of controlling them with light. ${ }^{13}$

\section{ACKNOWLEDGMENTS}

6 Nanostrukturen); the Alexander von Humboldt Stiftung 7 (Sofja Kovalevskaja Award), the European Research Council (ERC-StG 338258 "OptoQMol") and the Royal Society via the University Research Fellowship and URF - Grant.

We acknowledge financial support from the BadenWürttemberg Stiftung (Kompetenznetz Funktionelle

* corresponding author: lapo.bogani@materials.ox.ac.uk $\quad 61$

1 D. Gatteschi, R. Sessoli and J. Villain, Molecular Nano- 62 magnets, Oxford University Press (2006).

${ }^{2}$ G. E. Kostakis, I. J. Hewitt, A. M. Ako, V. Mereacre and 64 A. K. Powell, Philos. Trans. A. Math. Phys. Eng. Sci. 368, 65 1509-36 (2010).

3 R. W. Saalfrank, H. Maid and A. Scheurer, Angew. Chem. 67 Int. Ed. Engl. 47, 8794-824 (2008).

4 A. Mandel, W. Schmitt, T. G. Womack, R. Bhalla, R. K. 69 Henderson, S. L. Heath and A. K. Powell, Coord. Chem. 70 Rev. 190-192, 1067-1083 (1999).

${ }^{5}$ D. N. Woodruff, R. E. P. Winpenny and R. A. Layfield, 72 Chem. Rev. 113, 5110-48 (2013).

${ }^{6}$ K. S. Pedersen, J. Bendix and R. Clrac, Chem. Comm. 74 50, 4396-415 (2014).

7 R. Winpenny, Ed., Single-Molecule Magnets and Re- 76 lated Phenomena, Springer-Verlag, Berlin/Heidelberg, 77 112 (2006).

8 D. Gatteschi and R. Sessoli, Angew. Chem. Int. Ed. Engl. 79 42, 26897 (2003).

9 B. Barbara, L. Thomas, F. Lionti, I. Chiorescu and A. 81 Sulpice, J. Magn. Magn. Mater. 200, 167181 (1999). 82

${ }^{10}$ L. Gunther and B. Barbara, Quantum Tunneling of 83 Magnetization-QTM94: Proceedings of the NATO Ad- 84 vanced Research Workshop, Grenoble and Chichilianne, 85 France, June 27-July 2 1994, Kluwer Academic Pub 86 (1995).

11 J. R. Friedman, M. P. Sarachik, J. Tejada and R. Ziolo, 88 Phys. Rev. Lett. 76, 3830-3833 (1996).

12 W. Wernsdorfer and R. Sessoli, Science 284, 133-13590 (1999).

13 E. Heintze, F. El Hallak, C. Clauß, A. Rettori, M. G. Pini, F. Totti, M. Dressel and L. Bogani, Nat. Mater. 12, 202-6 93 (2013).

14 M. Slota, M. Blankenhorn, E. Heintze, M. Vu, R. Hbner and L. Bogani, Faraday Discussions 185, 347-359 (2015). 96

15 O. Sato, J. Tao and Y.-Z. Zhang, Angew. Chem. Int. Ed. 97 Engl. 46, 2152-87 (2007).

16 D. Pinkowicz, M. Ren, L.-M. Zheng, S. Sato, M. 99 Hasegawa, M. Morimoto, M. Irie, B. K. Breedlove, G.100 Cosquer, K. Katoh and M. Yamashita, Chemistry, 20,101 12502-13 (2014).

17 M. Verdaguer, Science 272, 698-699 (1996).

102

18 O. Sato, T. Iyoda, A. Fujishima and K. Hashimoto, Science 272, 704-705 (1996).

19 T. Liu, H. Zheng, S. Kang, Y. Shiota, S. Hayami, M. Mito,106 O. Sato, K. Yoshizawa, S. Kanegawa and C. Duan, Nat.107 Commun. 4, (2013).

20 S. Ohkoshi, S. Takano, K. Imoto, M. Yoshikiyo, A. Namai 109 and H. Tokoro, Nat. Photonics, 8, 65-71 (2013).
21 A. S. Zyazin, J. W. G. van den Berg, E. A. Osorio, H. S. J. van der Zant, N. P. Konstantinidis, M. Leijnse, M. R. Wegewijs, F. May, W. Hofstetter, C. Danieli and A. Cornia, Nano Lett., 10, 3307-11 (2010).

22 M. Trif, F. Troiani, D. Stepanenko and D. Loss, Phys. Rev. Lett. 101, 217201 (2008).

${ }^{23}$ O. Sato, T. Kawakami, M. Kimura, S. Hishiya, S. Kubo and Y. Einaga, J. Am. Chem. Soc. 126, 13176-7 (2004).

24 S.-I. Ohkoshi, K.-I. Arai, Y. Sato and K. Hashimoto, Nat. Mater. 3, 857-61 (2004).

25 S. Margadonna, K. Prassides and A. N. Fitch, Angew. Chem. Int. Ed. Engl. 43, 6316-9 (2004).

26 S. Maekawa, Concepts in spin electronics, Oxford University Press, 13 (2006).

27 J. Park, A. N. Pasupathy, J. I. Goldsmith, C. Chang, Y. Yaish, J. R. Petta, M. Rinkoski, J. P. Sethna, H. D. Abruña, P. L. McEuen and D. C. Ralph, Nature 417, 722-725 (2002).

28 D. Rugar, R. Budakian, H. J. Mamin and B. W. Chui, Nature 430, 329-32 (2004).

29 R. Vincent, S. Klyatskaya, M. Ruben, W. Wernsdorfer and F. Balestro, Nature 488, 357-60 (2012).

30 A. Saraiva-Souza, M. Smeu, L. Zhang, A. G. Souza Filho, H. Guo and M. A. Ratner, J. Am. Chem. Soc. 136, 1506571 (2014).

31 S. Sanvito, Chem. Soc. Rev. 40, 3336-55 (2011).

32 A. R. Rocha, V. M. Garca-Surez, S. W. Bailey, C. J. Lambert, J. Ferrer and S. Sanvito, Nat. Mater. 4, 335-9 (2005).

33 L. Bogani and W. Wernsdorfer, Nat. Mater. 7, 179-86 (2008).

34 S. Jiang, K. Go, C. Cervetti and L. Bogani, in Science China Chemistry 55, 867-882 (2012).

35 J. Choi, H. Lee, K. J. Kim, B. Kim and S. Kim, J. Phys. Chem. Lett. 1, 505-509 (2010).

36 S. Bhandary, S. Ghosh, H. Herper, H. Wende, O. Eriksson and B. Sanyal, Phys. Rev. Lett. 107, 257202 (2011).

37 A. Candini, S. Klyatskaya, M. Ruben, W. Wernsdorfer and M. Affronte, Nano Lett. 11, 2634-9 (2011).

38 H. G. Zhang, J. T. Sun, T. Low, L. Z. Zhang, Y. Pan, Q. Liu, J. H. Mao, H. T. Zhou, H. M. Guo, S. X. Du, F. Guinea and H.-J. Gao, Phys. Rev. B 84, 245436 (2011).

39 Y. Ying, R. K. Saini, F. Liang, A. K. Sadana and W. E. Billups, Org. Lett., 2003, 5, 14713.

40 S. Kyatskaya, J. R. G. Mascars, L. Bogani, F. Hennrich, M. Kappes, W. Wernsdorfer and M. Ruben, J. Am. Chem. Soc., 131, 15143-51 (2009).

41 L. Bogani, C. Danieli, E. Biavardi, N. Bendiab, A.-L. Barra, E. Dalcanale, W. Wernsdorfer and A. Cornia, Angew. Chemie, 121, 760-764 (2009). 
${ }^{42}$ L. Bogani and W. Wernsdorfer, Inorganica Chim. Acta, 64 2008, 361, 38073819.

43 H. P. Boehm, Carbon 32, 759-769 (1994).

44 L Routaboul, P Braunstein, J. Xiao, Z Zhang, P. A. ${ }^{6}$ Dowben, G. Dalmas, V. Da Costa, O. Flix, G. Decher, L. 68 G. Rosa and B. Doudin, J. Am. Chem. Soc. 134, 8494-506 69 (2012).

45 M. Mannini, F. Pineider, P. Sainctavit, C. Danieli, E. 71 Otero, C. Sciancalepore, A. M. Talarico, M.-A. Arrio, A. 72 Cornia, D. Gatteschi and R. Sessoli, Nat. Mater. 8, 194-7 73 (2009).

46 M. Mannini, F. Pineider, C. Danieli, F. Totti, L. Sorace, 75 P. Sainctavit, M.-A. Arrio, E. Otero, L. Joly, J. C. Cezar, 76 A. Cornia and R. Sessoli, Nature 468, 417-21 (2010). 77

47 C. F. Hirjibehedin, C.-Y. Lin, A. F. Otte, M. Ternes, C. 78 P. Lutz, B. A. Jones and A. J. Heinrich, Science 317, 79 1199-203 (2007).

48 A. Rajca, A. Olankitwanit, Y. Wang, P. J. Boratyski, M. 81 Pink and S. Rajca, J. Am. Chem. Soc. 135, 18205-15 82 (2013).

49 Y. Zhang, S. Kahle, T. Herden, C. Stroh, M. Mayor, U. 84 Schlickum, M. Ternes, P. Wahl and K. Kern, Nat. Com- 85 mun. 4, 2110 (2013).

50 C. Herrmann, G. C. Solomon and M. A. Ratner, J. Chem. 87 Phys., 2011, 134, 224306.

51 O. Kahn, Science 279, 44-48 (1998).

52 M. Maesato, T. Kawashima, Y. Furushima, G. Saito, H. 90 Kitagawa, T. Shirahata, M. Kibune and T. Imakubo, J. 91 Am. Chem. Soc. 134, 17452-5 (2012).

53 M. Mas-Torrent, N. Crivillers, V. Mugnaini, I. Ratera, C. 93 Rovira and J. Veciana, J. Mater. Chem.19, 1691 (2009). 94

${ }^{54}$ K. Matsuda and M. Irie, Chemistry 7, 3466-73 (2001). ${ }_{95}$

55 H. Nishide, S. Iwasa, Y.-J. Pu, T. Suga, K. Nakahara and 96 M. Satoh, Electrochim. Acta 50, 827-831 (2004). 97

56 W. Choi, D. Harada, K. Oyaizu and H. Nishide, J. Am. 98 Chem. Soc. 133, 19839-43(2011).

57 K. Nakahara, S. Iwasa, M. Satoh, Y. Morioka, J. Iriyama,100 M. Suguro and E. Hasegawa, Chem. Phys. Lett. 359, 351-101 354 (2002).

58 T. Suga, H. Ohshiro, S. Sugita, K. Oyaizu and H. Nishide,103 Adv. Mater. 21, 1627-1630 (2009).

59 K. Oyaizu and H. Nishide, Adv. Mater. 21, 2339-2344 105 (2009).

60 T Suga, H. Konishi and H. Nishide, Chem. Comm. 17302 (2007). L. Bugnon, C. J. H. Morton, P. Novak, J. Vetter and P.109 Nesvadba, Chem. Mater. 19, 2910-2914 (2007). 110

62 C. Simão, M. Mas-Torrent, N. Crivillers, V. Lloveras, J.111 M. Arts, P. Gorostiza, J. Veciana and C. Rovira, Nat.112 Chem. 3, 359364 (2011).

63 J. Lee, E. Lee, S. Kim, G. S. Bang, D. A. Shultz, R. D.114 Schmidt, M. D. E. Forbes and H. Lee, Angew. Chemie -115 Int. Ed. 50, 4414-4418 (2011).

64 I. Ratera and J. Veciana, Chem. Soc. Rev. 41, 303-49117 (2012).

65 A. Datcu, N. Roques, V. Jubera, D. Maspoch, X.19 Fontrodona, K. Wurst, I. Imaz, G. Mouchaham, J.-P.120 Sutter, C. Rovira and J. Veciana, Chemistry, 18, 152-62121 (2012).

66 C. Simão, M. Mas-Torrent, J. Veciana and C. Rovira,123 Nano Lett. 11, 4382-4385 (2011).

67 C. Herrmann, G. C. Solomon and M. A. Ratner, J. Am.125 Chem. Soc. 132, 3682-4 (2010).
${ }^{68}$ K. Sato, M. Yano, M. Furuichi, D. Shiomi, T. Takui, K. Abe, K. Itoh, A. Higuchi, K. Katsuma and Y. Shirota, J. Am. Chem. Soc. 119, 6607-6613 (1997).

69 K. Bernot, L. Bogani, A. Caneschi, D. Gatteschi and R. Sessoli, J. Am. Chem. Soc. 128, 7947-56 (2006).

${ }^{70}$ K. Bernot, J. Luzon, L. Bogani, M. Etienne, C. Sangregorio, M. Shanmugam, A. Caneschi, R. Sessoli and D. Gatteschi, J. Am. Chem. Soc. 131, 5573-9 (2009).

${ }^{71}$ K. E. Vostrikova, Coord. Chem. Rev. 252, 1409-1419 (2008).

72 C. Train, L. Norel and M. Baumgarten, Coord. Chem. Rev. 253, 2342-2351 (2009).

73 S. Haas, E. Heintze, S. Zapf, B. Gorshunov, M. Dressel and L. Bogani, Phys. Rev. B 89, 174409 (2014).

${ }^{74}$ L. Bogani, J. Appl. Phys. 109, 07B115 (2011).

75 M. Imada, A. Fujimori and Y. Tokura, Rev. Mod. Phys. 70, 1039-1263 (1998).

${ }^{76}$ K. Awaga and Y. Maruyama, J. Chem. Phys. 91, 2743 (1989).

77 A. Rose, Phys. Rev. 97, 1538-1544 (1955).

78 M. Lampert, Phys. Rev. 103, 1648-1656 (1956).

79 A. A. A. Grinberg, S. Luryi, M. R. Pinto and N. L. Schryer, IEEE Trans. Electron Devices 36, 1162-1170 (1989).

80 J. A. Geurst, Phys. status solidi 15, 107-118 (1966).

81 A. Ioannidis, E. Forsythe, Y. Gao, M. W. Wu and E. M. Conwell, Appl. Phys. Lett. 72, 3038 (1998).

${ }^{82}$ R. W. I. de Boer and A. F. Morpurgo, Phys. Rev. B 72, 073207 (2005).

${ }^{83}$ M. Stossel, J. Staudigel, F. Steuber, J. Blassing, J. Simmerer and A. Winnacker, Appl Phys Lett 76, 115-117 (2000).

84 G. Horowitz, Adv. Mater. 10, 365-377 (1998).

${ }^{85}$ E. F. Ullman, J. H. Osiecki, D. G. B. Boocock and R. Darcy, J. Am. Chem. Soc. 94, 7049-7059 (1972).

86 A. Caneschi, D. Gatteschi, R. Sessoli and P. Rey, Acc. Chem. Res. 22, 392-398 (1989).

87 E. T. Chernick, Q. Mi, R. F. Kelley, E. A. Weiss, B. A. Jones, T. J. Marks, M. A. Ratner and M. R. Wasielewski, J. Am. Chem. Soc. 128, 4356-64 (2006).

${ }^{88}$ K. Matsuda and M. Irie, J. Am. Chem. Soc. 122, 71957201 (2000).

${ }^{89}$ H. Komatsu, M. M. Matsushita, S. Yamamura, Y. Sugawara, K. Suzuki and T. Sugawara, J. Am. Chem. Soc. 132, 4528-9 (2010).

90 See Supplemental Material at [URL] for synthesis and structural data of the nitronyl-nitroxide radicals, further information on the statistical analysis on the transport measurements and a Mott-insulator scaling comparison with our data.

91 J. H. Osiecki and E. F. Ullman, J. Am. Chem. Soc. 90, 1078-1079 (1968).

92 E. F. Ullman, L. Call and J. H. Osiecki, J. Org. Chem. 35, 3623-3631 (1970).

93 W. Brtting, Phys. status solidi 201, 1035-1035 (2004).

94 M. Dressel and G. Grüner, Electrodynamics of Solids: Optical Properties of Electrons in Matter, Cambridge University Press (2002).

95 C. Cervetti, E. Heintze, B. Gorshunov, E. Zhukova, S. Lobanov, A. Hoyer, M. Burghard, K. Kern, M. Dressel, and L. Bogani, Adv. Mat. 27, 2676 (2015).

96 A. Baniodeh, Y. Liang, C. E. Anson, N. Magnani, A. K. Powell, A.-N. Unterreiner, S. Seyfferle, M. Slota, M. Dressel, L. Bogani and K. Goß, Adv. Funct. Mater. 24, 
6280-6290 (2014).

97 R. Smith and A. Rose, Phys. Rev. 97, 1531-1537 (1995). 56

98 R. W. I. de Boer, J. Appl. Phys. 95, 1196 (2004).

99 A. D. Schricker, F. M. Davidson, R. J. Wiacek and B. A. 58 Korgel, Nanotechnology 17, 2681-2688 (2006).

100 A. A. Talin, F. Léonard, B. S. Swartzentruber, X. Wang 60 and S. D. Hersee, Phys. Rev. Lett. 101, 076802 (2008). ${ }_{61}$

101 A. M. Katzenmeyer, F. Leonard, A. A. Talin, M. E. 62 Toimil-Molares, J. G. Cederberg, J. Y. Huang and J. L. 63 Lensch-Falk, IEEE Trans. Nanotechnol. 10, 9295 (1961). 64

102 G. T. Wright, Solid. State. Electron. 2, 165189 (1961). ${ }_{65}$

103 J. L. Brédas, J. P. Calbert, D. A. da Silva Filho and J. 66 Cornil, Proc. Natl. Acad. Sci. U. S. A., 99, 5804-5809 67 (2002).

104 W. Kohn, A. D. Becke and R. G. Parr, J. Phys. Chem., 69 1996, 100, 1297412980.

105 Y. Shao, L. F. Molnar, Y. Jung, J. Kussmann, C. Ochsen- 71 feld, S. T. Brown, A. T. B. Gilbert, L. V Slipchenko, S. V 72 Levchenko, D. P. ONeill, R. a DiStasio, R. C. Lochan, T. 73 Wang, G. J. O. Beran, N. a Besley, J. M. Herbert, C. Y. 74 Lin, T. Van Voorhis, S. H. Chien, A. Sodt, R. P. Steele, V. 75 a Rassolov, P. E. Maslen, P. P. Korambath, R. D. Adam- 76 son, B. Austin, J. Baker, E. F. C. Byrd, H. Dachsel, R. 77 J. Doerksen, A. Dreuw, B. D. Dunietz, A. D. Dutoi, T. 78 R. Furlani, S. R. Gwaltney, A. Heyden, S. Hirata, C.-P. 79 Hsu, G. Kedziora, R. Z. Khalliulin, P. Klunzinger, A. M. 80 Lee, M. S. Lee, W. Liang, I. Lotan, N. Nair, B. Peters, 81 E. I. Proynov, P. a Pieniazek, Y. M. Rhee, J. Ritchie, E. 82 Rosta, C. D. Sherrill, A. C. Simmonett, J. E. Subotnik, H. 83 L. Woodcock, W. Zhang, A. T. Bell, A. K. Chakraborty, 84 D. M. Chipman, F. J. Keil, A. Warshel, W. J. Hehre, H. 85 F. Schaefer, J. Kong, A. I. Krylov, P. M. W. Gill and 86 M. Head-Gordon, Phys. Chem. Chem. Phys. 8, 3172319187 (2006).

106 G. Kresse and J. Hafner, Phys. Rev. B 47, 558-561 (1993).

107 G. Kresse and J. Hafner, Phys. Rev. B 49, 14251-14269 90 (1994).

108 G. Kresse and J. Furthmüller, Comput. Mater. Sci. 6 15-50 (1996)

109 G. Kresse and J 11186 (1996)

110 G. Kresse and D. Joubert, Phys. Rev. B 59, 1758 (1999). 96

111 J. P. Perdew, K. Burke and M. Ernzerhof, Phys. Rev. 97 Lett. 77, 3865 (1996).

112 H. J. Monkhorst and J. D. Pack, Phys. Rev. B 13, 5188- 99 5192 (1976).

113 V. Coropceanu, J. Cornil, D. A da Silva Filho, Y Olivier, R. Silbey and J.-L. L. Brèdas, Chem. Rev. 107, 926-952102 (2007).

114 I. G. Austin and N. F. Mott, Adv. Phys. 18, 41-102(1969).104

115 N. N. Dinh, L. H. Chi, T. T. Chung Thuy, T. Q. Trung 105 and V.-V. Truong, J. Appl. Phys. 105, 093518 (2009). ${ }_{106}$

116 H.-S. Woo, Y.-B. Kim, R. Czerw, D. L. Carroll, J. Ballato and P. M. Ajayan, J. Korean Phys. Soc.45, 507-511
(2004).

117 S. M. Sze and K. K. Ng, Physics of Semiconductor Devices, John Wiley \& Sons, Inc., Hoboken, NJ, USA, 3 (2006).

118 N. Karl, Synthetic Metals 133, 649-657 (2003).

119 P. Mark and W. Helfrich, J. Appl. Phys. 33, 205 (1962).

120 P. E. Burrows, Z. Shen, V. Bulovic, D. M. McCarty, S. R. Forrest, J. A. Cronin and M. E. Thompson, J. Appl. Phys. 79, 7991 (1997).

121 A. J. Campbell, D. D. C. Bradley and D. G. Lidzey, J. Appl. Phys. 82, 6326 (1997).

122 M. A. Lampert and P. Mark, Current injection in solids, Academic Press, (1970).

123 W. Brtting, S. Berleb and A. G. Mückl, Org. Electron. 2, 136 (2001).

124 P. W. M. Blom, M. J. M. de Jong and J. J. M. Vleggaar, Appl. Phys. Lett.,68, 3308 (1996).

125 J. E. Anthony, Chem. Rev. 106, 5028-48 (2006).

126 Y. Shirota and H. Kageyama, Chem. Rev. 107, 953-1010 (2007).

127 B. Purushothaman, M. Bruzek, S. R. Parkin, A.-F. Miller and J. E. Anthony, Angew. Chemie, 123, 7151-7155 (2011).

128 M. E. Gershenson and V. Podzorov, Rev. Mod. Phys. 78 973-989 (2006).

129 S. A. DiBenedetto, A. Facchetti, M. A. Ratner and T. J. Marks, Adv. Mater. 21, 1407-1433 (2009).

130 V. Podzorov, E. Menard, A. Borissov, V. Kiryukhin, J. A. Rogers and M. E. Gershenson, Phys. Rev. Lett. 93, 086602 (2004).

131 V. A. Dediu, L. E. Hueso, I. Bergenti and C. Taliani, Nat. Mater. 8, 707-716 (2009).

132 V. Dediu, M. Murgia, F. C. C. Matacotta, C. Taliani and S. Barbanera, Solid State Commun. 122, 181-184 (2002).

133 F. J. J. Wang, Z. H. H. Xiong, D. Wu, J. Shi and Z. V. V Vardeny, Synth. Met. 155, 172-175 (2005).

134 C. Barraud, P. Seneor, R. Mattana, S. Fusil, K. Bouzehouane, C. Deranlot, P. Graziosi, L. Hueso, I. Bergenti, V. Dediu, F. Petroff and A. Fert, Nat. Phys. 6, 615-620 (2010).

135 Z. H. Xiong, D. Wu, Z. V. Vardeny and J. Shi, Nature 427, 821-4 (2004).

136 C. Cervetti, A. Rettori, M. G. Pini, A. Cornia, A. Repolls, F. Luis, M. Dressel, S. Rauschenbach, K. Kern, M. Burghard and L. Bogani, Nature materials doi:10.1038/nmat4490.

137 L. Bogani, R. Maurand, L. Marty, C. Sangregorio, C. Altavilla, and W. Wernsdorfer, J. Mat. Chem. 20, 2099-2107 (2010).

138 L. Bogani, Experiments on Molecular Magnets for Molecular Spintronics, Structure and Bonding, Springer, Berlin, Germany, 164, 331-381 (2014). 